\title{
Yrast line for weakly interacting trapped bosons
}

\author{
George F. Bertsch and Thomas Papenbrock \\ Institute for Nuclear Theory, Department of Physics, University of Washington, Seattle, WA \\ 98195, USA
}

\begin{abstract}
We compute numerically the yrast line for harmonically trapped boson systems with a weak repulsive contact interaction, studying the transition to a vortex state as the angular momentum $L$ increases and approaches $N$, the number of bosons. The $L=N$ eigenstate is indeed dominated by particles with unit angular momentum, but the state has other significant components beyond the pure vortex configuration. There is a smooth crossover between low and high $L$ with no indication of a quantum phase transition. Most strikingly, the energy and wave function appear to be analytical functions of $L$ over the entire range $2 \leq L \leq N$. We confirm the structure of low- $L$ states proposed by Mottelson, as mainly single-particle excitations with two or three units of angular momentum.
\end{abstract}

PACS numbers: 03.75.Fi, 05.30.Jp, 67.40.Db 
The low-lying excitations of atomic Bose Einstein condensates in harmonic traps [1 3] are of considerable experimental and theoretical interest [4]. Recently, Mottelson proposed a theory for the yrast line of weakly interacting $N$-boson systems [5], i.e. the ground states at nonvanishing angular momentum $L$. Physical arguments led him to assume that the yrast states are excited upon acting on the ground state $|0\rangle$ of vanishing angular momentum with a collective operator $Q_{\lambda}=\sum_{p=1}^{N} z_{p}^{\lambda}$ that is a sum of single-particle operators acting on the coordinates $z_{p}=x_{p}+i y_{p}$ of the $p^{\text {th }}$ particle. For angular momenta $L \ll N$ the yrast states are found to be dominated by quadrupole $(\lambda=2)$ and octupole $(\lambda=3)$ modes. Assuming a vortex structure of the yrast states with $L \approx N$ then led to the prediction of a quantum phase transition in Fock space when passing from the low angular momentum regime $L \ll N$ to the regime of high angular momenta $L \approx N$. The reason for this is behavior is the approximate orthogonality of the collective states $Q_{\lambda}|0\rangle$ and the singleparticle oscillator states of the vortex line in the regime $N^{1 / 2} \ll L$. These results have been obtained for harmonically trapped bosons with a weak repulsive contact interaction. The case of an attractive interaction has been studied by Wilkin et al. [6]. In this case the total angular momentum is carried by the center of mass motion, and there are no excitations corresponding to relative motion. This is not unexpected since internal excitations would increase the energy of the yrast state.

It is the purpose of this letter to present an independent numerical computation of the yrast line and to compare with Mottelson's results [5]. In particular we want to focus on the transition from low to high angular momentum yrast states. The investigation of this transition is of interest not only for the physics of Bose-Einstein condensates. Localization in Fock space is under investigation also in molecular [7] and condensed matter physics [8,9]. The numerical computation has the advantage that it does not rely on the assumptions made in the analytical calculation. However, with our numerical methods it is limited to angular momenta below about $\mathrm{E} \approx 50$. Most interestingly, our numerical results suggest that the yrast line and the corresponding wave functions can be presented by rather simple analytical expressions. 
Let us consider $N$ bosons in a two-dimensional harmonic trap interacting via a contact interaction'? We are interested in the yrast line in the perturbative regime of weak interactions. Note however that experimental studies of trapped condensates are often in a regime where the interaction energy is comparable to the trapping potential, and this may introduce qualitatively different physics. We write the Hamiltonian as

$$
\hat{H}=\hat{H}_{0}+\hat{V} \text {. }
$$

Here

$$
\hat{H}_{0}=\hbar \omega \sum_{j} j \hat{a}_{j}^{\dagger} \hat{a}_{j}
$$

is the one-body oscillator Hamiltonian and

$$
\hat{V}=g \sum_{i, j, k, l} V_{i j k l} \hat{a}_{i}^{\dagger} \hat{a}_{j}^{\dagger} \hat{a}_{k} \hat{a}_{l} .
$$

the two-body interaction. The operators $\hat{a}_{m}$ and $\hat{a}_{m}^{\dagger}$ annihilate and create one boson in the single-particle oscillator state $|m\rangle$ with energy $m \hbar \omega$ and angular momentum $m \hbar$, respectively and fulfill bosonic commutation rules. The ground state energy is set to zero. Up to some irrelevant overall constant the matrix elements are given by $V_{i j k l}=2^{-k-l}(k+l) ! /(i ! j ! k ! l !)^{1 / 2}$ and vanish for $i+j \neq k+l$. For total angular momentum $L$ the Fock space is spanned by states $|\alpha\rangle \equiv\left|n_{0}, n_{1}, \ldots, n_{k}\right\rangle$ with $\sum_{i=0, k} n_{i}=N, \hat{a}_{j}^{\dagger} \hat{a}_{j}\left|n_{0}, n_{1}, \ldots, n_{k}\right\rangle=n_{j}\left|n_{0}, n_{1}, \ldots, n_{k}\right\rangle$ and $\sum_{j=0, k} j n_{j}=L$. Here $n_{j}$ denotes the occupation of the $j^{\text {th }}$ single particle state $|j\rangle$. For vanishing coupling $g$ the basis states are degenerate in energy, and the problem thus consists in diagonalizing the two-body interaction $\hat{V}$ inside the Fock space basis. To set up the matrix we act with the operator (3) onto one initial basis state with angular momentum $L$ and onto all states created by this procedure until the the Fock space is exhausted [10]. The resulting matrix is sparse, and the yrast state is computed using a Lanczos algorithm [11]. We restrict ourselves to $L \leq 50$ corresponding to a maximal Fock space dimension of about $d_{L} \approx 2 \cdot 10^{5}$.

\footnotetext{
${ }^{1}$ The results obtained below extend to the three dimensional problem for $L=L_{z}$.
} 
The yrast line, i.e. the ground state energies as a function of the angular momentum may be written as $E(L)=L \hbar \omega+g \epsilon_{L}$. Fig. 1 shows the $L$-dependence of the energies $\epsilon_{L}$ for systems of $N=25$ and 50 bosons. The energies $\epsilon_{L}$ simply decrease linearly with increasing angular momentum for $L \leq N$. In fact to machine precision, the energy function is found to described by an algebraic expression,

$$
\epsilon_{L}=\frac{N(2 N-L-2)}{2}
$$

At fixed angular momentum $L$ and for $L \ll N$ the energies $g \epsilon_{l}$ increase as expected with the square of the number of bosons $N$. Notice in the figure that there is a kink in the slope at $N=L$. This is a hint of condensation into a vortex state: in macroscopic superfluids, the state for $L=N$ would have a condensate of unit angular momentum and would be lower in energy than neighboring yrast states.

We next investigate the structure of the wave functions of yrast states. We would like to know how complex the states are and how well they can be described by single-particle operators acting on simple states. To address the question of the complexity of the states in the Fock basis, we take the wave function amplitudes $c_{\alpha}^{(L)}$ in the Fock representation of the state

$$
|L\rangle=\sum_{\alpha=1}^{d_{L}} c_{\alpha}^{(L)}|\alpha\rangle
$$

and compute the inverse participation ratio [12

$$
I_{L} \equiv \sum_{\alpha=1}^{d_{L}}\left|c_{\alpha}^{(L)}\right|^{4} .
$$

The $I_{L}$ is the first nontrivial moment of the distribution of wave function intensities $\left|c_{\alpha}^{(L)}\right|^{2}$. Its inverse $1 / I_{L}$ measures the number of basis states $|\alpha\rangle$ that have significant overlap with the yrast state $|L\rangle$. Fig 2 shows a plot of $1 / I_{L}$ and the Fock space dimension $d_{L}$ as a function of angular momentum $L$ for a system of $N=50$ bosons. The $1 / I_{L}$ is seen to be much smaller than the dimensionality of the Fock space. Even where the participating is greatest, at midvalues of $L$, only about 30 states are active participants. A similar behavior of quantum non-ergodicity has been found previously in numerical studies [10]. Notice that the inverse 
participation ratio decreases strongly as $N=L$ is approached. This shows that the yrast state becomes simpler, again hinting at the formation of a vortex condensate. Examining the coefficients for $N=25$ in detail, the largest amplitude at $L=N$ is in fact the vortex state, $|\alpha\rangle=\mid 0 N 0 \ldots 0>$, but it has less than half the probability of the complete wave function. Interestingly, our numerically obtained yrast state $|L=N=25\rangle$ agrees with the conjecture given by Wilkin et al. [6], i.e. $|L=N\rangle=\prod_{p=1}^{N}\left(z_{p}-z_{c}\right)|0\rangle$ with $z_{c}=N^{-1} \sum_{p=1}^{N} z_{p}$ being the center of mass. Based on our numerical wave functions, we can generalize this conjecture. We believe that all the yrast states for $2 \leq L \leq N$ are given by the formula

$$
|L\rangle=\sum_{p_{1}<p_{2}<\ldots<p_{L}}\left(z_{p_{1}}-z_{c}\right)\left(z_{p_{2}}-z_{c}\right) \ldots\left(z_{p_{L}}-z_{c}\right) \mid 0>
$$

We have verified that this formula is correct (up to machine precision) by comparison with the numerically obtained yrast states for $N=25$. Since the operator acting on the ground state is translationally invariant no quanta of the center of mass motion are excited. Notice that there is a natural termination of the construction at $L=N$.

To further examine the structure of the yrast states $|L\rangle$ we show a plot of the occupation numbers $n_{j}^{(L)} \equiv\left\langle L\left|\hat{a}_{j}^{\dagger} \hat{a}_{j}\right| L\right\rangle$ for $j=0,1,2,3$ in Fig. 3 for a system of $N=50$ bosons. At very low angular momenta the yrast states are dominated by single particle oscillator states with two or three units of angular momentum. This is in agreement with Mottelson's results [5]. However, at larger angular momentum $L$ the dominant fraction is carried by single particle states with one unit of angular momentum. Note that the occupation numbers $n_{j}^{(L)}$ are very small for $j>3$. This analysis confirms the results found for the inverse participation ratio. Note also that the observables $n_{j}^{(L)}$ are very smooth functions of $L$. If there were a quantum phase transition at large $L \approx N / 2$, we would expect to see some precursor in these observables.

In conclusion, our numerical study strongly indicates that there is no quantum phase transition to a vortex state for trapped condensates in the limit that the interaction potential is small compared to the oscillator frequency. The strongest evidence is the apparent existence of analytic expressions for the energies and the wave functions on the yrast line for 
$2 \leq L \leq N$ One might speculate that these states are contained in a dynamical symmetry group, but we have no idea how this might come aboutf. We have also examined the structure of the yrast states and the matrix elements between them, finding that the observables vary smoothly with $L$, for $L$ not too small.

We acknowledge conversations with B. Mottelson. This work was supported by the Dept. of Energy under Grant DE-FG-06-90ER40561.

\footnotetext{
${ }^{2}$ We note also that there is another symmetry group [13], $S O(2,1)$, that produces relationships between energies of different states within a single $L$ subspace.
} 


\section{REFERENCES}

[1] M. N. Anderson, J. R. Ensher, M. R. Matthews, C. E. Wieman, and E. A. Cornell, Science 269, 198 (1995)

[2] C. C. Bradley, C. A. Sacket, J. J. Tollet, and R. G. Hulet, Phys. Rev. Lett. 75, 1687 (1995)

[3] K. B. Davis, M.-O. Mewees, M. R. Andrews, N. J. van Druten, D. S. Durfee, D. M. Kurn, and W. Ketterle, Phys. Rev. Lett. 75, 3969 (1995)

[4] For a review see, e.g., F. Dalfovo, S. Giorgini, L. P. Pitaevskii, and S. Stringari, Rev. Mod. Phys. 71, 463 (1999)

[5] B. Mottelson, e-print cond-mat/9905053

[6] N. K. Wilkin, J. M. Gunn, and R. A. Smith, Phys. Rev. Lett. 80, 2265 (1998)

[7] D. M. Leitner and P. G. Wolynes, Chem. Phys. Lett. 258, 18 (1996)

[8] B. L. Altshuler, Y. gefen, A. Kamenev, and L. S. Levitov, Phys. Rev. Lett. 78, 2803 (1997)

[9] C. Mejía-Monasterio, J. Richert, T. Rupp, and H. A. Weidenmüller, Phys. Rev. Lett. 81, $5189(1998)$

[10] T. Papenbrock and G. F. Bertsch, Phys. Rev. A 58, 4854 (1998)

[11] R. B. Lehoucq, D. C. Sorensen, and Y. Yang, ARPACK User's Guide: Solution to large scale eigenvalue problems with implicitly restarted Arnoldi methods, FORTRAN code available under http://www.caam.rice.edu/software/ARPACK/

[12] L. Kaplan, Nonlinearity 12, R1 (1999)

[13] L.P. Pitaevshii and A. Rosch, Phys. Rev. A55 R853 (1998). 


\section{FIGURES}

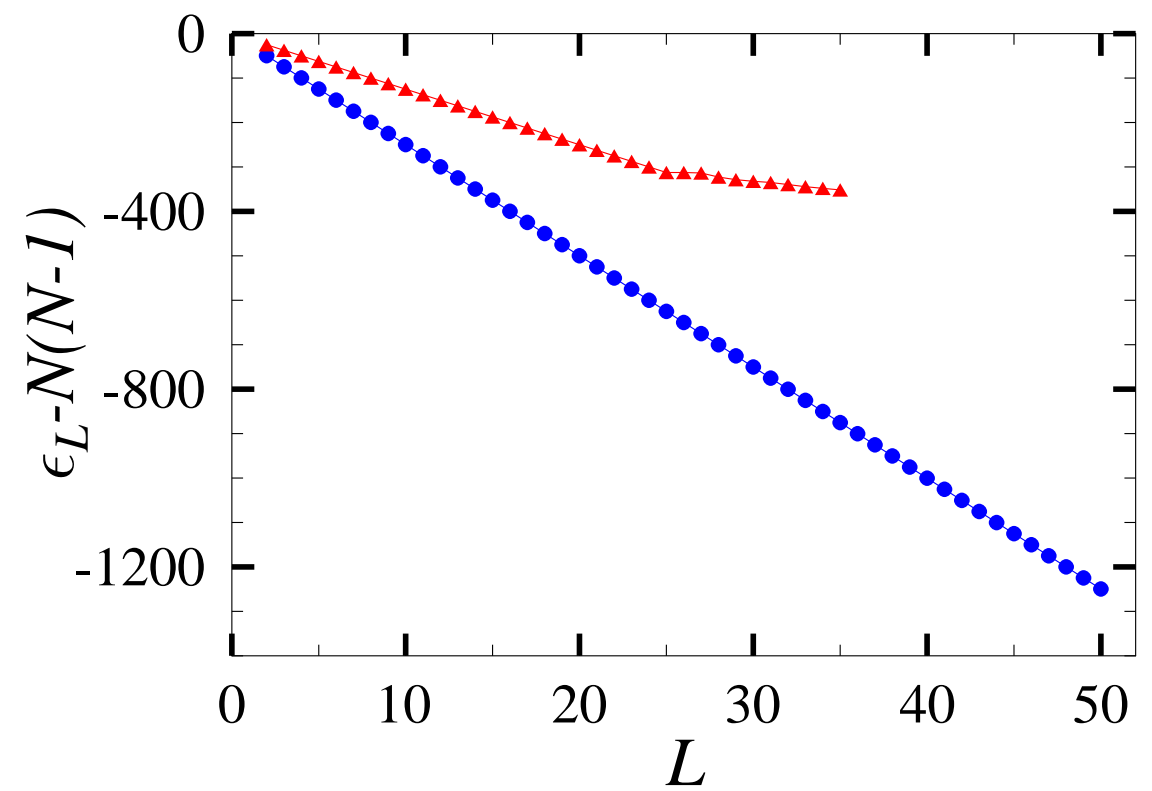

FIG. 1. Interaction energy $\epsilon_{L}$ as a function of angular momentum $L$ for systems of $N=50$ (circles) and $N=25$ bosons (triangles).

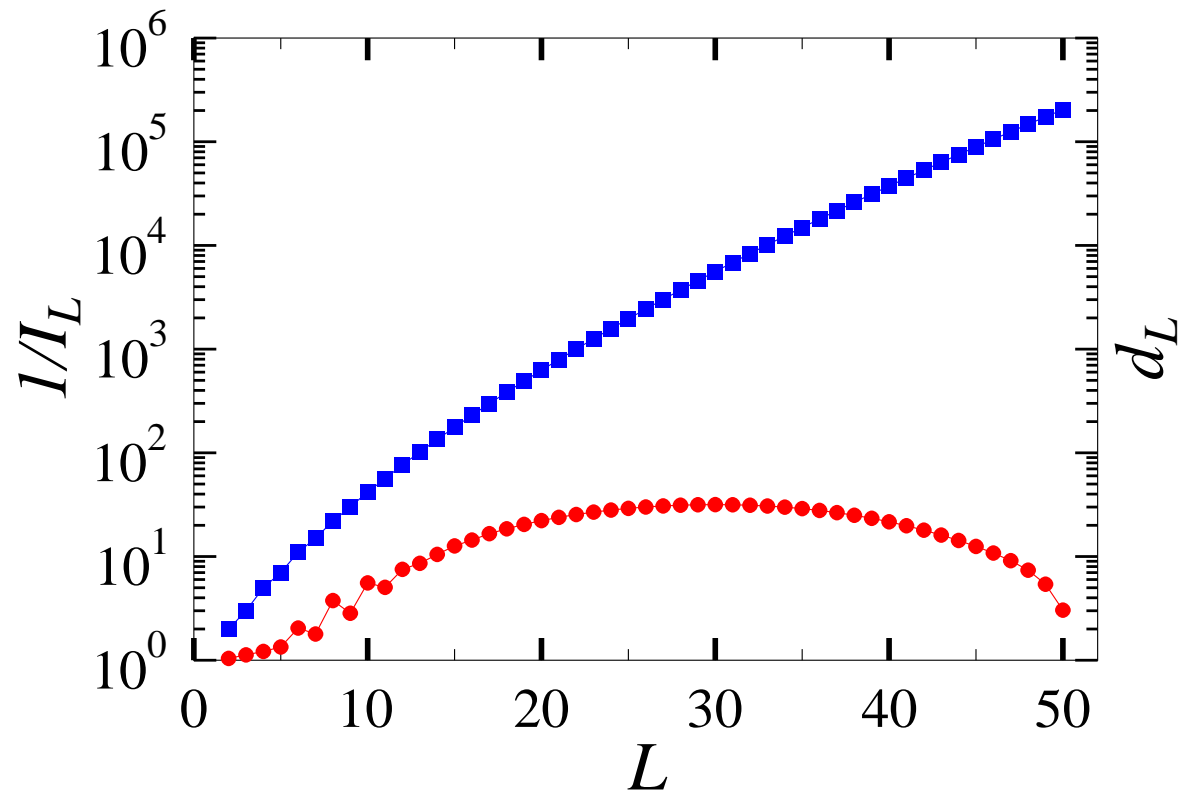

FIG. 2. Inverse participation ratio $1 / I_{L}$ (circles) and Fock space dimension $d_{L}$ (squares) as a function of angular momentum $L$ for a system of $N=50$ bosons. 


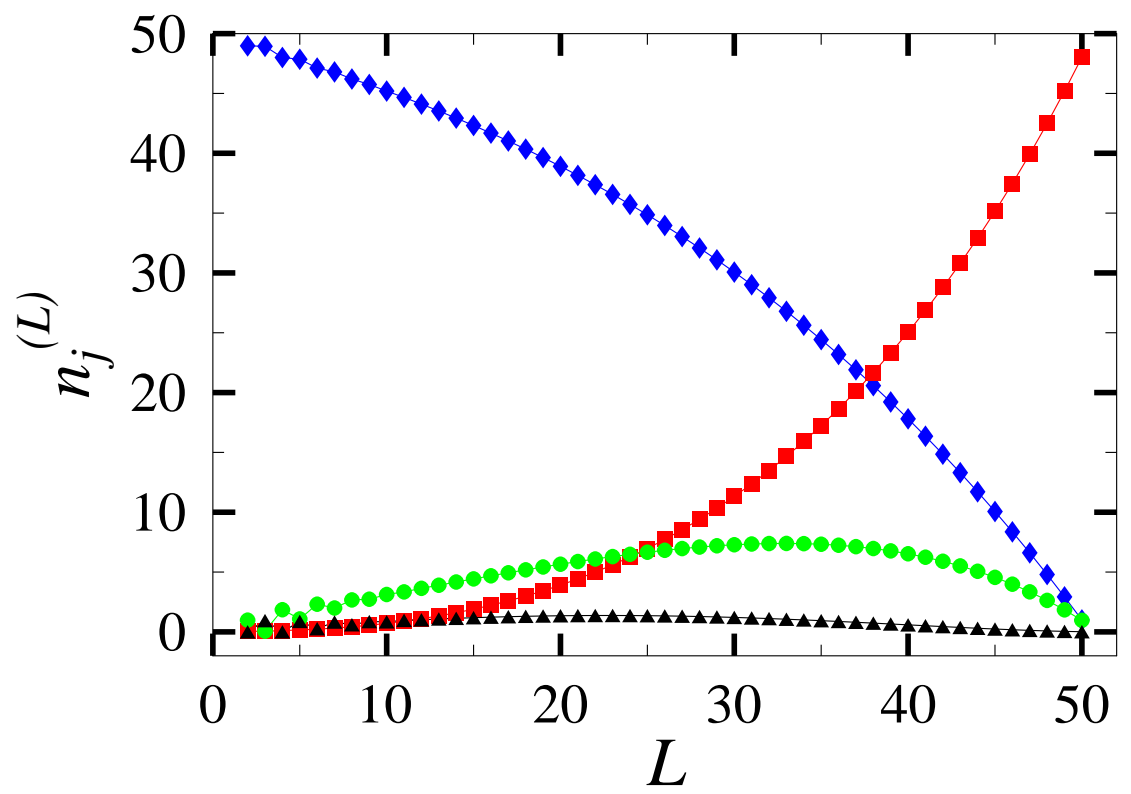

FIG. 3. Occupation numbers of the lowest single particle oscillator states as a function of angular momentum $L$ for a system of $N=50$ bosons. $(j=0$ : diamonds, $j=1$ : squares, $j=2$ circles, $j=3$ triangles) 